\title{
Photo-induced growth of silver nanoparticles using UV sensitivity of cellulose fibers
}

\author{
A. Alahgholipour Omrani ${ }^{\text {a,* }}$, N. Taghavinia ${ }^{b, c}$ \\ a Institute of Condensed Matter Physics, Ecole Polytechnique Fédérale de Lausanne, CH-1015 Lausanne, Switzerland \\ b Physics Department, Sharif University of Technology, Tehran 14588, Iran \\ ' Institute for Nanoscience and Nanotechnology, Sharif University of Technology, Tehran 14588, Iran
}

\section{A R T I C L E I N F O}

\section{Article history:}

Received 15 September 2011

Received in revised form 6 October 2011

Accepted 7 October 2011

Available online 2 November 2011

\section{Keywords:}

Silver

Nanoparticles

UV irradiation

Cellulose

\begin{abstract}
A B S T R A C T
A simple method has been demonstrated to grow silver nanoparticles on the surface of cellulose fibers. The preparation is based on photo-activation of surface by ultraviolet (UV) photons, followed by chemical reduction of silver nitrate. It is found that the concentration of silver nitrate in the solution is not a determining factor, while UV intensity affects the rate of initial growth and determines the final concentration of the loaded silver. We explain the phenomena based on a model including the number of reducing sites on the surface of cellulose fibers activated by UV photons, and a release mechanism that causes a slow rate of dissolution of silver back into the solution.
\end{abstract}

(c) 2011 Elsevier B.V. All rights reserved.

\section{Introduction}

In the last few decades, there has been increased interest in antibacterial coatings on textile materials because of a variety of environmental pollutants. The use of organic antibacterial agents, such as halogen compounds having aromatic groups, poses safety concerns and is being evaded. Silver has been known as a disinfectant for many years and is being used in many forms in the treatment of infectious diseases [1-3]. Silver ions, even in very low concentrations, show a broad spectrum of antibacterial activity, while exhibiting low toxicity towards mammalian cells [4,5]. Silver nanoparticles, having a high ratio of surface to volume, are active as antibacterial agents if they are coated on the surface of materials, such as textile fibers.

Silver nanoparticles have been synthesized by either direct evaporation of silver into a liquid medium, such as laser ablation [6-9], or by reducing silver ions chemically [10-12] or through irradiation by electrons [13], ultraviolet (UV) light [14] or Gamma ray $[15,16]$. To load silver nanoparticles on the surface of fibers, the fibers are usually exposed to a silver nanoparticle sol, resulting in random attachment of nanoparticles on the surface [17-19]. The problem in this case is the weak binding of nanoparticles on the surface, so that after a few times washing they are completely removed. There are also reports on electrospinning of

\footnotetext{
* Corresponding author. Tel.: +41 21693 4144; fax: +41 216934666

E-mail address: arash.omrani@epfl.ch (A.A. Omrani).
}

polymers containing silver ions and reducing agents. Silver ions will be reduced on the surface and polymer fibers containing silver nanoparticles are formed [20,21]. UV irradiation has also been used to initiate the reduction by activating a sensitive agent. However, in these cases some unwanted precursors will finally remain in the matrix.

Here we employ cellulose fibers both as a support and as a sensitive agent to form $\mathrm{Ag}$ nanoparticles on the surface. $\mathrm{Ag}^{+}$ions are reduced on the surface of cellulose fibers under UV irradiation. In this case, the shortcomings of the other methods are weaker, as the nanoparticles will be firmly attached on the surface and precursors will not remain in the matrix after washing. UV photon absorption is expected to break the oxygen bridge between the glucose monomers, forming aldehyde groups that can reduce $\mathrm{Ag}^{+}$ ions $[22,23]$. We will give a qualitative model to explain the growth mechanism.

\section{Experimental}

Silver nitrate powder was supplied by Merck Co. Germany, and used without further purification. Cotton textile as pure cellulose fibers has been used in this work. Deionized (DI) water was employed throughout the work. An $8 \mathrm{~W}$ low pressure mercury lamp with a dominant radiation peak at $254 \mathrm{~nm}$ was used as the UV source.

In a typical procedure, $5 \mathrm{~cm} \times 5 \mathrm{~cm}$ cotton textile used as cellulose fibers was immersed in $30 \mathrm{ml} 0.015 \mathrm{M} \mathrm{AgNO}_{3}$ solution in a glass plate and irradiated using an $8 \mathrm{~W}$ UV lamp at a distance 

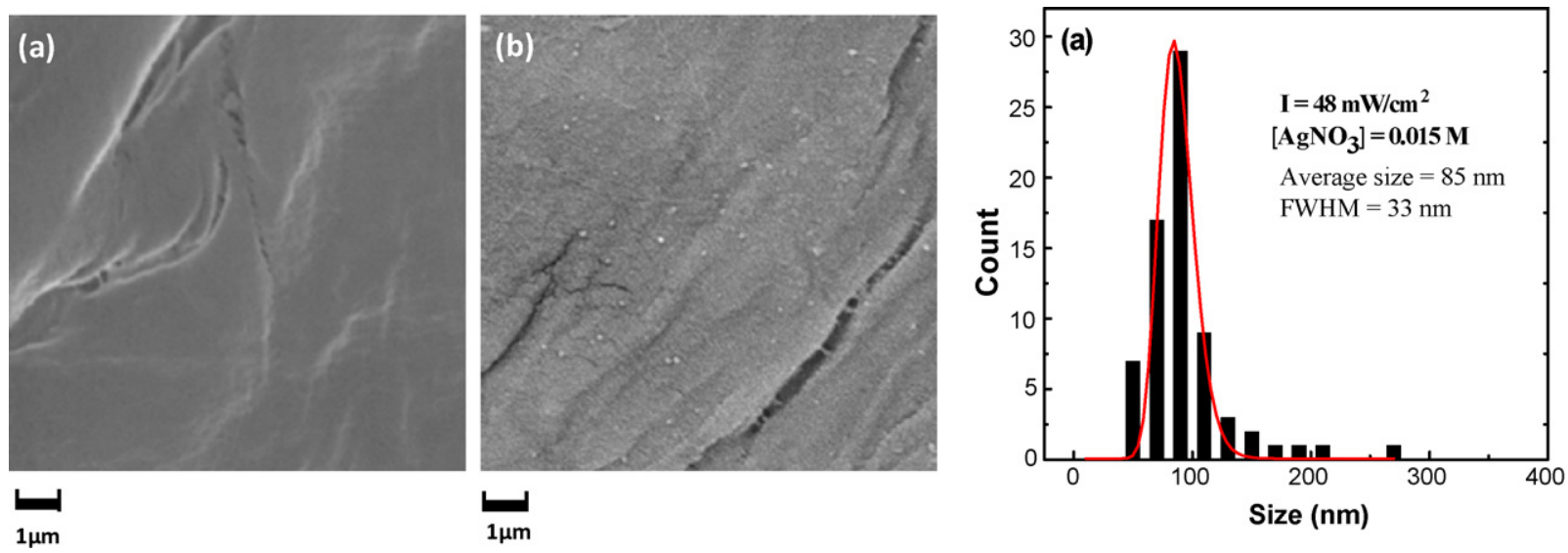

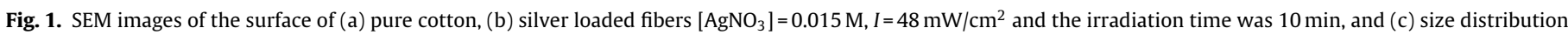
of silver nanoparticles.

of $10 \mathrm{~cm}$. In some experiments $\mathrm{AgNO}_{3}$ concentration and UV irradiation intensity were changed. UV intensity on the fibers was controlled by changing the lamp-sample distance. As the fiber surface was activated by UV irradiation, fibers took various colors from bright yellow to dark brown, depending on the experimental conditions. The samples were finally washed with DI water and dried in air at room temperature. It is important to use DI water, as silver nitrate remaining in the textile is very sensitive to existing ions in water, in particular chloride ions which cause the formation of $\mathrm{AgCl}$ precipitates.

Scanning Electron Microscopy (SEM) was made using a Philips XL30 machine. Diffused Reflectance Spectroscopy (DRS) measurements of samples were made by AvaSpec-2048TEC spectrometer. The same spectrometer was used to measure the absolute UV intensity at the sample surface. The composition of the surface could be determined by X-ray photoelectron spectroscopy (XPS). Measurements were carried out in a BESTEC instrument calibrating the binding energies (BE) to the $\mathrm{Au} \mathrm{f}_{7 / 2}$ level taken as $84.0 \mathrm{eV}$.

\section{Results and discussion}

\subsection{Formation of silver nanoparticles}

Fig. 1 shows typical SEM images of the surface of fibers demonstrating the formation of silver nanoparticles on the surface due to UV irradiation. The white spots represent the silver islands formed on the surface, as verified by XPS wide-scan spectrum of cellulose after UV irradiation beside silver nitrate, which is shown in Fig. 2. $\mathrm{The} A \mathrm{~g}(3 \mathrm{~d})$ peaks refer to the silver nanoparticle not to the silver ions because samples washed after preparation so there is not any silver ions on the sample surface. The SEM images demonstrate that silver deposition on the surface has proceeded through the formation of scattered silver nanoparticles rather than a layered coating. The particles are uniformly distributed on the surface.

\subsection{The effect of UV irradiation intensity and solution concentration on the growth process}

SEM images were employed to obtain the size and size distribution of nanoparticles. Typical graphs which qualitatively show the effect of UV intensity as well as $\mathrm{AgNO}_{3}$ concentration are shown in Fig. 3. The graphs were fitted using log-normal functions. As illustrated in the figure, the size distribution of nanoparticles is rather broad. The size distribution graphs of a number of samples with different irradiation times show that the distribution width (full width at half maximum, FWHM) increased with the average size and in many cases was almost equal to the average size. The broad distribution may imply that nucleations have been formed at different times during the growth process.

Fig. $3 a$ and $b$ illustrates the effect of the UV intensity on the size distribution for samples which were irradiated for $10 \mathrm{~min}$. The average size for UV intensities of $40 \mathrm{~mW} / \mathrm{cm}^{2}$ and $48 \mathrm{~mW} / \mathrm{cm}^{2}$ were $56 \mathrm{~nm}$ and $85 \mathrm{~nm}$, respectively. The increase in the average size by increasing the UV intensity is expected; however, as shown later, this is not a linear relation. Also comparing Fig. 3b and c qualitatively, the effect of $\mathrm{AgNO}_{3}$ concentration on the size distribution is explored. Here the average size of particles slightly increases by using ten times more concentrated $\mathrm{AgNO}_{3}$. Hence, the size is not much affected by the solution concentration.

Fig. 4 shows how the average size is increased with irradiation time, for samples of Fig. 3. While there is an increasing trend with time, one observes that the size growth is not much dependent on $\mathrm{AgNO}_{3}$ concentration at times longer than about $5 \mathrm{~min}$. This implies that most of the size growth takes place before $5 \mathrm{~min}$, and later the size tends to saturation, in particular after $20 \mathrm{~min}$.

Fig. 5 displays the absorption spectra of the samples using DRS measurements. The peak at around $437 \mathrm{~nm}$ refers to surface plasmon resonance (SPR) of silver nanoparticles. The position and shape of SPR peak is known to depend on the size and shape of nanoparticles [24,25], as well as the effective dielectric constant of the medium $[26,27]$. Here the peak position increases very slowly with

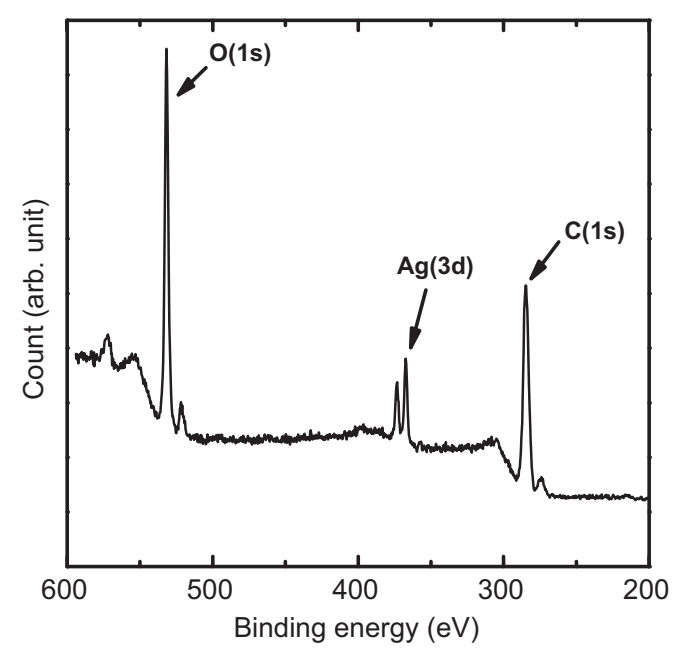

Fig. 2. XPS diagram for a sample prepared by $0.015 \mathrm{M} \mathrm{AgNO}_{3}, 40 \mathrm{~mW} / \mathrm{cm}^{2}$ intensity and 80 min irradiation time. 

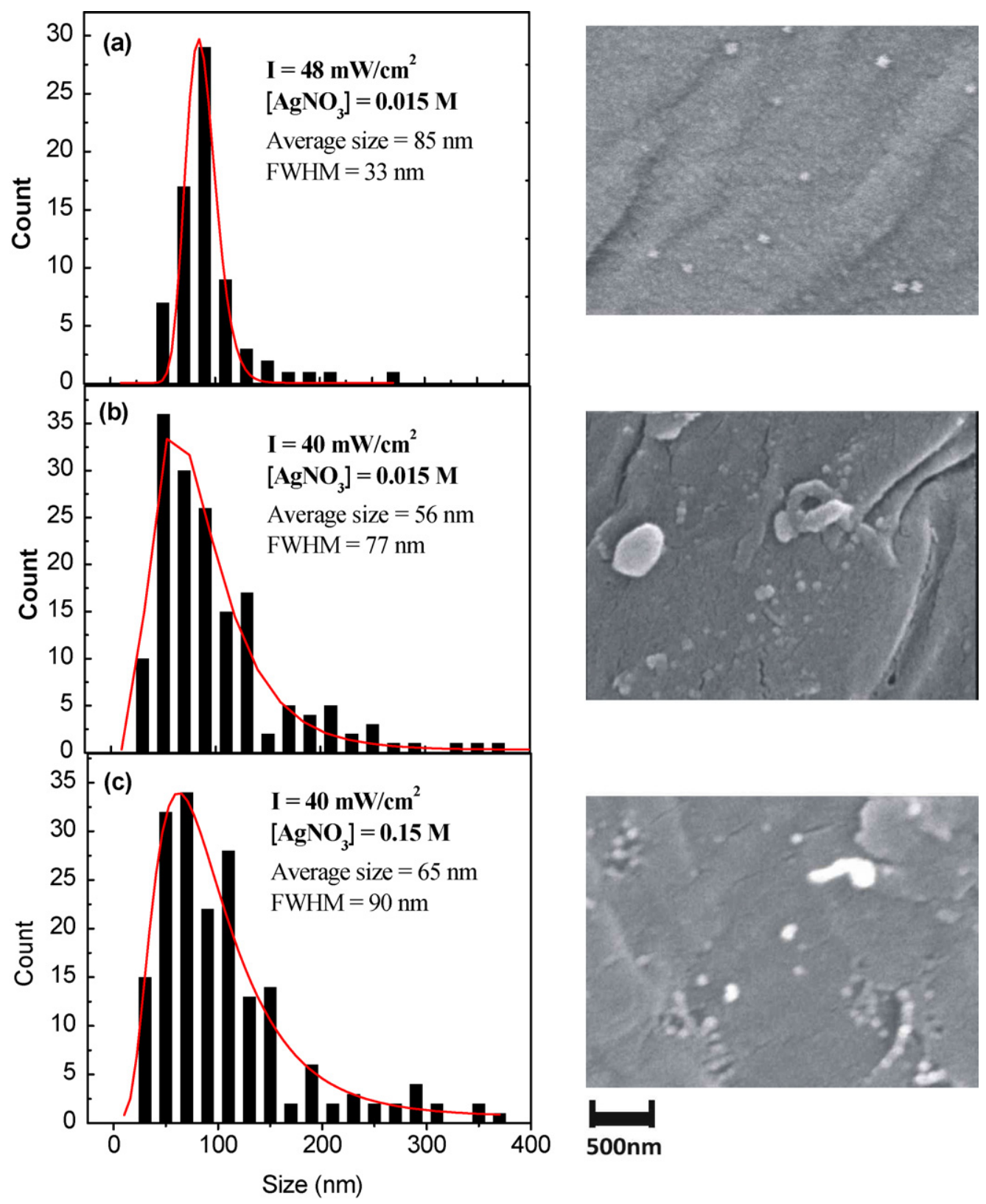

$500 \mathrm{~nm}$

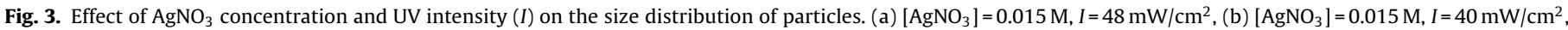
and (c) $\left[\mathrm{AgNO}_{3}\right]=0.15 \mathrm{M}, I=40 \mathrm{~mW} / \mathrm{cm}^{2}$, the irradiation time was $10 \mathrm{~min}$.

irradiation time, hence the size of silver nanoparticles does not largely increase.

The absorption peaks show an extended tail at longer wavelengths. The tail is not observed for free spherical silver nanoparticles synthesized in solution $[25,28]$. The origin of the tail could relate to non-spherical shape of the particles, wide size distribution or heterogeneity of the medium around the particles, as explained by Gans models [29]. The SEM observations show the particles shape does not deviate much from spherical shape. Also, the size broadening, though high in our case, cannot broaden the SPR peaks to this extent. Therefore, it is very probable that the tail of the SPR peaks originate from the variation of the dielectric constant of the medium surrounding the particles. We have observed by X-ray photoelectron spectroscopy (XPS) that the surface of nanoparticles is partially oxidized. In addition, each particle is surrounded by a different fraction of air and cellulose, depending on the location of the particle on cellulose fiber surface. This may explain why the SPR peaks are broadened into long wavelengths.

The height of the absorption peaks in Fig. 5 is proportional to the concentration of the silver loaded on the fibers. Fig. 6 displays the peak heights versus the irradiation time for the absorption peaks of Fig. 5, as well as samples with other UV intensities. It is observed that by increasing the irradiation time initially the peak height rises very fast and then turns to a saturation mode, that is, silver is first rapidly formed on the surface, while at longer times no more silver is added on the surface. The rise time differs for different UV intensities, however $20 \mathrm{~min}$ can be regarded as a rough estimate. Fig. 6 also verifies that UV intensity can influence the slope of the initial rise, as well as the saturation trend. Higher UV intensity results in faster increase of the peak height and earlier saturation. Also the saturation value increases with UV intensity; i.e. more reduced Ag exist on the cellulose fibers irradiated with higher UV intensities. 


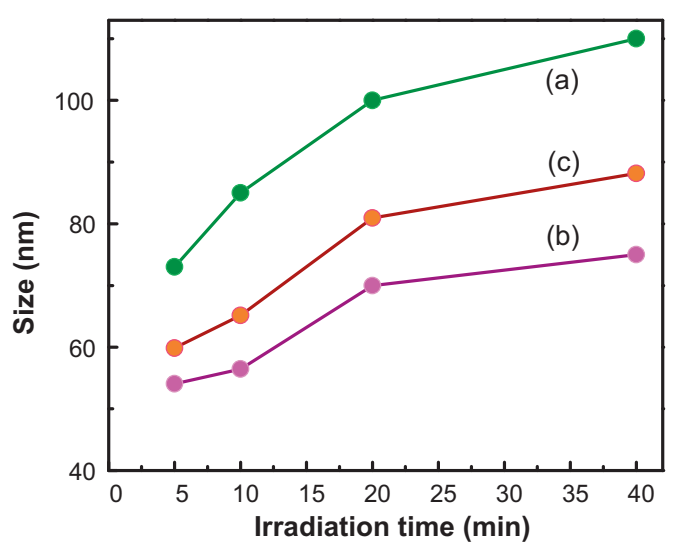

Fig. 4. Changes of average particles size versus different irradiation times. (a) $\left[\mathrm{AgNO}_{3}\right]=0.015 \mathrm{M}, I=48 \mathrm{~mW} / \mathrm{cm}^{2}$, (b) $\left[\mathrm{AgNO}_{3}\right]=0.015 \mathrm{M}, I=40 \mathrm{~mW} / \mathrm{cm}^{2}$, and (c) $\left[\mathrm{AgNO}_{3}\right]=0.15 \mathrm{M}, I=40 \mathrm{~mW} / \mathrm{cm}^{2}$, the irradiation time was $10 \mathrm{~min}$.

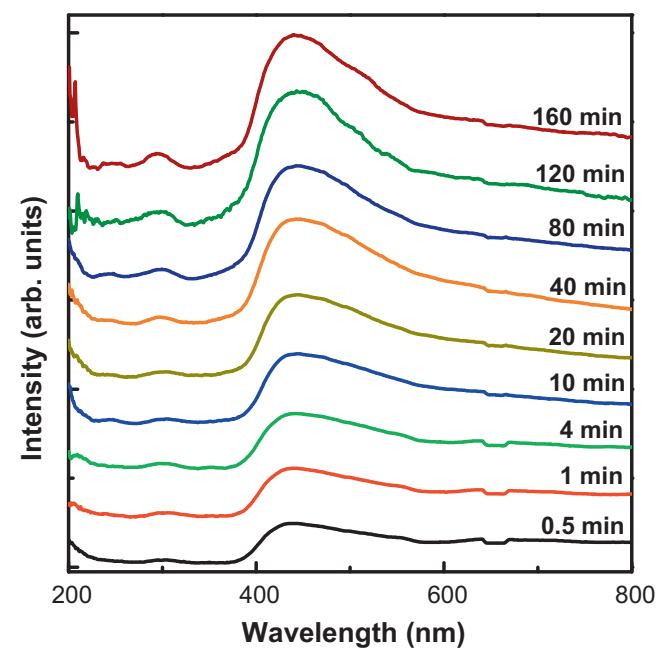

Fig. 5. DRS diagrams for the samples prepared by $0.015 \mathrm{M} \mathrm{AgNO} 3,40 \mathrm{~mW} / \mathrm{cm}^{2}$. Intensity and different irradiation times are written on the graphs.

Fig. 7 illustrates the time evolution of growth for various $\mathrm{AgNO}_{3}$ concentrations. One observes that in the beginning of the growth process there is not any dependence to the solution concentration. The growth saturates on longer times, however the dependence of the saturation conditions on the solution concentration is very weak, compared to the effect of UV intensity. We have noticed that the cause of the growth saturation was not the depletion of the $\mathrm{Ag}^{+}$ions in the solution. To verify this, the same solution was used to prepare several samples in a sequence. It was observed, using DRS data, that the concentration of silver loaded on the samples

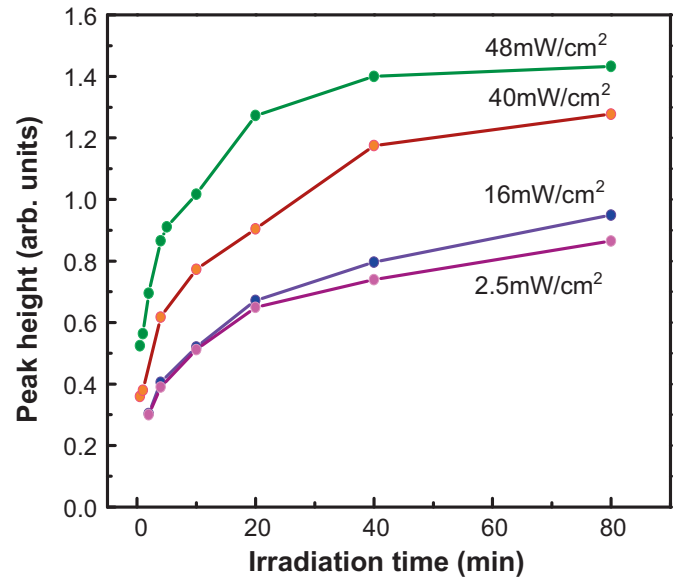

Fig. 6. The time evolution of the height of SPR peaks for different UV intensities The values are proportional to the amount of silver loaded on the fibers.

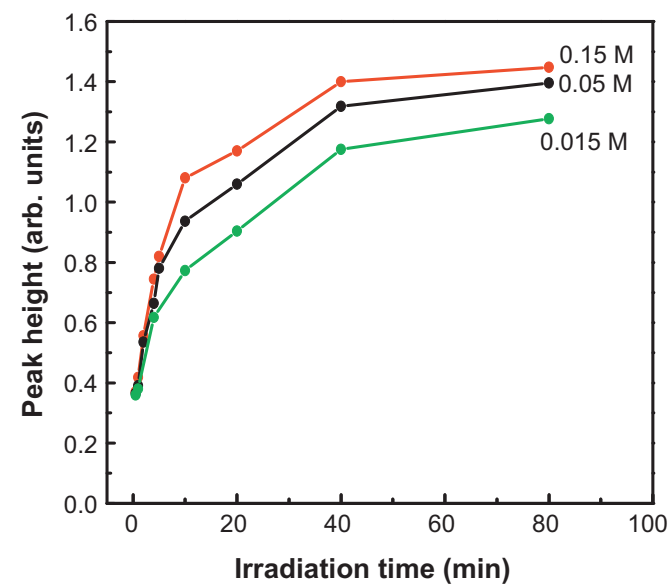

Fig. 7. The time evolution of the height of surface plasmon peaks for different solution concentrations.

was only slightly different. Similar trend of growth in Fig. 7 also demonstrates that different solution concentrations cannot lead to a difference in growth saturation.

\subsection{Mechanism for growth of silver nanoparticles}

The data in Figs. 6 and 7 demonstrate that UV intensity affects the initial growth rate, as well as the saturation level, however, the $\mathrm{AgNO}_{3}$ concentration makes no considerable effect. In a simple model to understand these observations one can assume that there is a certain number of sites on a unit area of the surface of

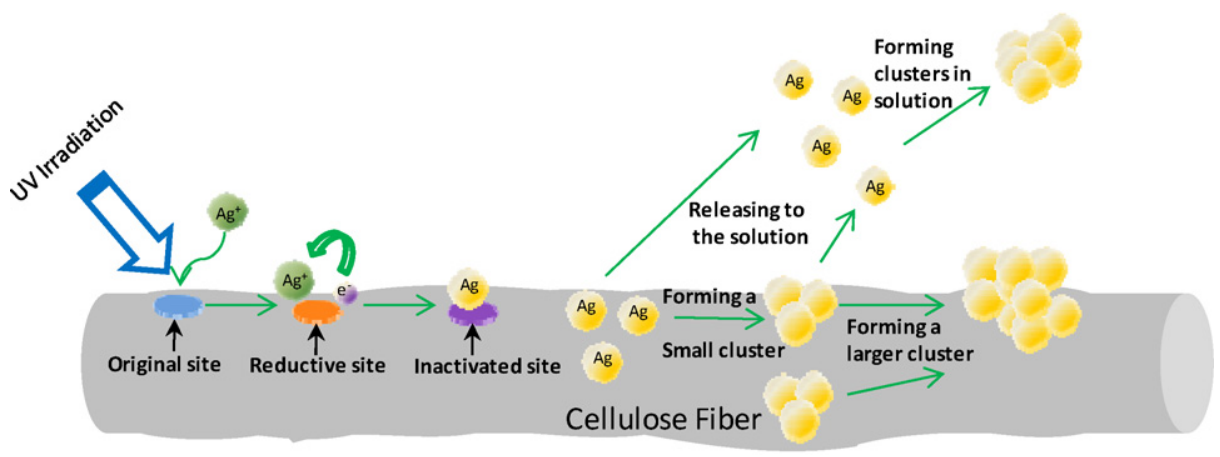

Scheme 1. A schematic model representing the process of silver growth on the surface of cellulose fibers. 
cellulose fibers that can turn reducing upon UV photon absorption. Each site might be a cellulose monomer (a glucose ring) that forms aldehyde groups upon photon absorption [30,31]. When cellulose is irradiated by UV, the oxygen bonding between the glucose rings will break and form an aldehyde agent that could reduce $\mathrm{Ag}^{+}$ions. These aldehyde agents change to a carboxyl group after reduction. For this reason the activated sites are expected to be permanently de-activated after they reduce an $\mathrm{Ag}^{+}$ion. With this picture of the process, one should expect faster rate of Ag formation with increasing UV intensity due to faster photon absorption by sites on the surface. This is observed in Fig. 6. It is also expected that the same amount of $\mathrm{Ag}$ is formed on the surface due to number of active sites can form on the surface, if sufficient time is given, regardless of UV intensity. This is not confirmed by data in Fig. 6, which shows that more Ag is formed on the surface by higher UV intensities. Scheme 1 is a qualitative representation of the growth model. A small amount of $\mathrm{Ag}$ release into the solution was also experimentally observed, as the solution turned slightly yellow and showed small SPR peak of $\mathrm{Ag}$, especially for longer irradiation times.

\section{Conclusion}

Ag nanoparticles can be grown on the surface of cellulose fibers by activating them with UV photons. It was observed that $\mathrm{AgNO}_{3}$ concentration plays not a determining role in the final concentration of silver on the fibers, while UV intensity is an important factor in the growth. The active sites on fiber surface determine the capacity of the surface for reducing silver. Based on the presented model, there is a certain concentration of sites on the surface, which acquires reducing property by UV excitation, and then permanently de-activated after reducing $\mathrm{Ag}^{+}$ions. It was observed that more $\mathrm{Ag}$ is formed on the surface for higher UV intensities.

\section{References}

[1] G. Zhao, E. Stevens, Biometals 11 (1998) 27

[2] F. Fu-Ren, A. Bard, J. Phys. Chem. B 106 (2002) 279.

[3] R. Demling, L. deSanti, Wounds 13 (2001) 11.

[4] R.L. Williams, P.J. Doherty, D.G. Vince, G.J. Grashoff, D.F. Williams, Crit. Rev. Biocompat. 5 (1989) 221.

[5] T.J. Berger, J.A. Spadaro, S.E. Chapin, R.O. Becker, Antimicrob Agents Chemother. 9 (1976) 357.

[6] I. Lee, S.W. Han, K.J. Kim, J. Raman Spectrosc. 32 (2001) 947.

[7] R. Brause, H. Moltgen, K. Kleinermanns, Appl. Phys. B-Lasers Opt. 75 (2002) 711.

[8] Y.H. Chen, C.S. Yeh, Colloids Surf., A 197 (2002) 133.

[9] T. Tsuji, N. Watanabe, M. Tsuji, Appl. Surf. Sci. 211 (2003) 189.

[10] D.L. Van Hyning, C.F. Zukoski, Langmuir 14 (1998) 7034

[11] Y.D. Yin, Z.Y. Li, Z.Y. Zhong, B. Gates, Y.N. Xia, S. Venkateswaran, J. Mater. Chem. $12(2002) 522$.

[12] P.C. Lee, D. Meisel, J. Phys. Chem. 86 (1982) 3391.

[13] K.A. Bogle, S.D. Dhole, V.N. Bhoraskar, Nanotechnology 17 (2006) 3204.

[14] S. De, A. Pal, N.R. Jana, A. Pal, J. Photochem. Photobiol. A 131 (2000) 111.

[15] A. Henglein, Langmuir 17 (2001) 2329.

[16] S.H. Choi, S.H. Le, Y.M. Hwang, K.P. Lee, H.D. Kang, Radiat. Phys. Chem. 67 (2003) 517.

[17] H.J. Lee, S.Y. Yeo, S.H. Jeong, J. Mater. Sci. 38 (2003) 2199.

[18] H.J. Lee, S.H. Jeong, Textile Res. J. 74 (5) (2004) 442-452.

[19] S.Y. Yeo, H.J. Lee, S.H. Jeong, J. Mater. Sci. 38 (2003) 2143.

[20] Q.B. Yang, D.M. Li, Y.L. Hong, Z.Y. Li, C. Wang, S.L. Qiu, Y. Wei, Synth. Met. 137 (2003) 973.

[21] H.K. Lee, E.H. Jeong, C.K. Baek, J.H. Youk, Mater. Lett. 59 (2005) 2977.

[22] N.E. Kotelnikova, G. Wegener, T. Paakkari, R. Serimaa, V.N. Demidov, A.S. Serebriakov, A.V. Shchukarev, A.V. Gribanov, Russ. J. Gen. Chem. 73 (3) (2003) 418-426.

[23] N.E. Kotelnikova, V.N. Demidov, G. Wegener, E. Windeisen, Russ. J. Gen. Chem. 73 (3) (2003) 427-433.

[24] Y. Zhao, Y. Jiang, Y. Fang, Spectrochim. Acta A 65 (2006) 1003.

[25] J.J. Mock, M. Barbic, D.R. Smith, D.A. Schultz, S. Schultz, J. Chem. Phys. 116 (15) (2002) 6755-6759.

[26] S.K. Mandal, R.K. Roy, A.K. Pal, J. Phys. D: Appl. Phys. 35 (2002) 2198

[27] S.K. Mandal, R.K. Roy, A.K. Pal, J. Phys. D: Appl. Phys. 36 (2003) 261.

[28] S. Kapoor, Langmuir 14 (1998) 1021.

[29] V.M. Renteria, J. Garcia-Macedo, Colloids Surf., A 278 (2006) 1.

[30] R.A. Stillings, R.J. Van Nostrand, J. Am. Chem. Soc. 66 (1944) 753.

[31] G.S. Egerton, J. Soc. Dyers Colour. 65 (1949) 764. 